\title{
Das Recht auf den Tod
}

\section{Seraphina Limacher}

Medizinstudentin, Universität Zürich

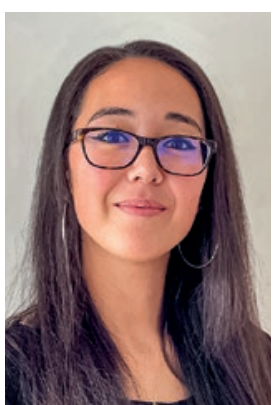

Blaulicht, Sirenen und ein Abschiedsbrief. Ein 14-jähriges Mädchen wird mit reduziertem Allgemeinzustand, Benommenheit und einer Medikamentenintoxikation in die Notaufnahme des Kinderspitals eingeliefert. Trotz Schläfrigkeit sagt sie mit bestimmter Stimme: «Lasst mich sterben.» Als das Mädchen aufgefunden wurde, waren haufenweise Medikamente in ihrer Tasche enthalten. Pflegefachkräfte, Ärztinnen und Ärzte versammeln sich im Behandlungszimmer. Das Standardprozedere bei Vergiftungen wird eingeleitet, es werden Telefonate mit dem Tox-Zentrum geführt, Zugänge gelegt, die panischen Eltern empfangen und über die Situation aufgeklärt. Doch immer wieder zwischendurch ihre leise Stimme: «Ich will sterben. Hört auf, euch um mich zu sorgen!» Die Laborwerte sind da. Zusammen mit den Ergebnissen der körperlichen Untersuchung sehen die Umstände nicht gut aus. Es werden abnormale Herz- und Atemgeräusche festgestellt, ihr Puls ist stark erhöht, und das Blutbild weist bereits auf eine potenzielle Leberschädigung hin. Trotz ihrer wiederholten Bitte, alle Massnahmen zu unterlassen, lautet das Vorgehen: Gabe eines Antidots und eine Gastroduodenoskopie mit Magenspülung, um die verbleibenden Reste der Medikamente zu entfernen. Zusätzlich wird intra- und postoperativ in regelmässigen Abständen Aktivkohle verabreicht, damit weitere Schadstoffe gebunden werden können.

Der Arztbericht, auf den sich dieses Fallbeispiel stützt, stammt vom 22.10.2012. Ich rechne nach und stelle fest, dass das Mädchen, welches sich da versucht hat, das Leben zu nehmen, gleich alt ist wie ich. Anbei der Abschiedsbrief, der sich Wort für Wort in mein Gedächtnis gebrannt hat. Als Medizinstudentin im ersten Jahr ist es schwierig für mich, mir überhaupt vorzustellen, was damals in den Köpfen des Ärzte- und Pflegeteams vorgegangen ist. An die Situation der hilflosen Eltern möchte ich gar nicht denken, wie enorm schwer die Angst und Ungewissheit auf ihren Schultern lastete. Für die Eltern ist das Vorgehen in diesem Moment klar, die Rettung ihrer Tochter steht im Vordergrund. Doch auf den ersten Blick können wir oft nicht erkennen, wie vielschichtig eine Entscheidungsfindung ist, vor die sich die Fachleute gestellt sehen. Als Laie scheinen die Kriterien festgesetzt zu sein durch unsere Vorstellung, wie Ärztinnen und Ärzte oder Pflegefachpersonen handeln sollen und müssen oder welchen Pflichten sie in ihrem Beruf nachgehen. Doch für das ungeschulte Auge sind die vier Grundsätze nach Beauchamp und Childress nicht ersichtlich, welche immer wieder eine wichtige Rolle in der Medizin spielen. Die vier Prinzipien Respekt vor der Autonomie, Wohltun, Nicht-Schaden und Gerechtigkeit.

Ein schwer greifbares Konzept, da sich in der Bevölkerung die Ansicht eingebürgert hat, dass medizinische Fachkräfte «nur» die Rettung und Genesung ihrer Patientinnen und Patienten verfolgen und um jeden Preis Heilung ermöglichen wollen. Sobald man sich aber mit der ethischen Ansicht in der Medizin befasst, wird einem bewusst, wie komplex die Behandlung einer Patientin bzw. eines Patienten sein kann und dass die angeblich leichte Entscheidung über das Verfahren nur als Fassade dient für ein allgegenwärtiges Dilemma. So ergeht es momentan auch mir. Während der Aufarbeitung des obengenannten Falls stosse ich immer wieder an Konfliktpunkte, die sich die verschiedenen Prinzipien liefern. Hier sind es vor allem die Autonomie, das Prinzip des Wohlwollens und des Nicht-Schadens, die ich nicht miteinander in Einklang bringen kann, egal wie herum ich die Situation drehe. Jede alternative Möglichkeit bringt mich zum gleichen Ergebnis: Man muss sich entscheiden. Entscheiden, welcher Grundsatz weniger gewichtet wird.

Mein Entsetzen über das Schicksal des jungen Mädchens vereint sich nach und nach mit Neugierde und Faszination, meine Liebe zur Medizin und zur Philosophie entfaltet sich, und ich befinde mich, ehe ich mich versehe, in vielen ethisch, metaphysisch angehauchten Fragen über das Warum und Wieso der Vorgehensweise der Ärzte und Pflegefachpersonen.

Ich frage mich, wie die Situation wäre, wenn es sich bei der eingelieferten Patientin nicht um ein 14-jähriges Schulmädchen handeln würde, sondern um einen erwachsenen Mann mit der gleichen Ausgangslage. Wäre in diesem Fall ebenfalls die Behandlung im Vordergrund und würde man dementsprechend dem Patienten trotz deutlicher Bitte nach dem Tod das Recht zu sterben "verweigern»? Die Autonomie des Mädchens wurde bewusst übergangen und ihr die Entscheidung über ihr eigenes Abtreten abgenommen. Schliesslich war sie zu diesem Zeitpunkt minderjährig, unmündig und nur bedingt urteilsfähig. Diese Kriterien scheinen aber im fiktiven Fall, den ich mir in meinem Kopf 
zusammenbastelte, alle erfüllt zu sein. Jedoch macht mich ein Begriff stutzig. Ein wenig paradox, in Suizidfällen von Urteilsfähigkeit zu sprechen, nicht? Sie lässt sich nicht nur anhand des physischen Zustands einer Person festlegen, sondern erfordert ebenfalls einen Einblick in das psychische Befinden. Nicht nur geistige Behinderungen, sondern auch mentale Krankheiten wie beispielsweise Depression führen dazu, dass die Urteilsfähigkeit eines Menschen angezweifelt werden kann. Dies, weil nicht ersichtlich ist, ob Suizidversuche oder Selbstverletzungen ein Ausdruck der Person selbst sind oder sich diese Verhaltensmuster durch die psychische Labilität manifestiert haben.

Frustration macht sich in mir breit, denn ich merke, dass sich ebenfalls in meinem Denken über die vergangenen zwei Jahrzehnte die pflichtengeprägte Ansicht des Arztberufs verankert hat, die ich wohl oder übel spätestens nach diesem Essay endgültig ablegen muss. Die Reichweite der ärztlichen Aufgaben nimmt ein völlig anderes Ausmass an. Einerseits gilt es, der Patientin bzw. dem Patienten die bestmögliche Versorgung mit den vorhandenen Mitteln zu gewährleisten, andererseits aber auch den Wunsch des vor einem liegenden Menschen zu respektieren. Wieder einmal Wohltun gegen Autonomie.

Widmet man sich der Pflichtenethik, kommt man um Immanuel Kant nicht herum. Der deutsche Philosoph prägte diesen spezifischen Bereich der Ethik mit dem kategorischen Imperativ. Dieser besagt, dass man nach denjenigen «Maximen» zu handeln hat, die uneingeschränkt gelten sollen. Taten stützen sich auf das Pflichtbewusstsein und geschehen ohne Eigenvorteil. Zudem gilt der Grundsatz: "Handle so, dass du die Menschheit sowohl in deiner Person, als in der Person eines jeden anderen jederzeit zugleich als Zweck, niemals bloss als Mittel brauchest» [1]. - Sowohl in deiner Person, die Pflicht gilt also auch sich selbst gegenüber. Zum Thema Suizid hatte Kant eine ganz klare Ansicht, was mich zum eigentlichen Punkt meiner Gedankengänge bringt: Sie verstösst gegen jegliche moralischen und ethischen Konzepte. Dies nicht, weil er als streng religiöser Mensch das Leben als heilig und Geschenk Gottes ansah, welches als unantastbar galt, sondern eben genau wegen der oben genannten Selbstzweckformel. Man zerstört sich selber, entflieht somit zwar der misslichen Lebenslage, in der man sich zuvor gefangen sah, verwendet aber sein eigenes Ich nur als Mittel zum Zweck zur Erlangung der eigenen Glückseligkeit. Eine nach Kant egoistisch gewertete Handlung, welche sich aber heutzutage immer noch stark widerspiegelt im gesellschaftlichen Gedankengut. Nach wie vor sind psychische Störungen, selbstverletzendes Verhalten und Suizid weit tabuisierte Probleme, behaftet mit vielen Stigmatisierungen der Opfer. Selbstmord wird noch heute als ichbezogen und rücksichtslos bezeichnet, die Hintergründe bleiben oft unverstanden oder nicht beachtet.

Kein Mensch möchte in der Haut der behandelnden Ärztin bzw. des behandelnden Arztes stecken, welcher in diesem Moment tatsächlich gewissermassen Gott spielt und das weitere Leben (oder gegebenenfalls den Tod) einer anderen Person in der Hand hält. Ein klares Handlungsschema gemäss den vier medizinethischen Prinzipien zu erstellen ist eine Sache der Unmöglichkeit, denn in jeder Dilemma-Situation muss nicht nur die objektive Faktenbasis beachtet werden, sondern auch die subjektive, mentale Verfassung der Person. Vor allem sind die Behandlungspfade und Genesungschancen in solchen aussergewöhnlichen Notfallsituationen nicht ersichtlich, der Blick in die Zukunft bleibt einem verwehrt. Dies erschwert die Beurteilung der Lage zusätzlich, und es kann nicht immer im Vornherein gesagt werden, ob dieser spezifische Patient bzw. die Patientin in Zukunft froh ist, dass man seinen bzw. ihren innigsten Wunsch damals überging und die lebensrettenden Massnahmen einleitete. Körperliche und seelische Heilung erfordern einen starken Willen und die Bereitschaft, auch Rückfälle auf sich zu nehmen. Wenn ein Mensch dazu nicht bereit oder in der Lage ist, dann ist der nächste Schritt herauszufinden, wie die verbleibende Zeit, seien es Stunden, Tage oder Monate, so angenehm und schmerzlos wie möglich zu gestalten ist. Die Würde des Patienten bzw. der Patientin zu wahren hat dabei oberste Priorität, selbst wenn das bedeuten mag, dass man gegen die eigenen oder gesellschaftlich vertretenen moralischen und ethischen Grundsätze verstösst. Schweren Herzens muss man sich doch als (zukünftige) Ärztin eingestehen, dass nicht jede Patientin bzw. jeder Patient zu retten ist. Ärzte und Ärztinnen sind keine Halbgötter in Weiss und können ihren Patientinnen und Patienten nicht immer die vollständige körperliche und seelische Heilung garantieren.

Ich betrachte mich im Spiegel und versuche krampfhaft nachzuvollziehen, was in diesem Mädchen aus dem Fallbeispiel vorgegangen ist. Tränen füllen meine Augen und mein Spiegelbild verschwimmt, zu schmerzhaft ist der Gedanke daran, dass ein solch junges Mädchen sich an einem Punkt in seinem Leben wiederfindet, an dem der scheinbar einzige Ausweg der Freitod ist. Wo befindet sie sich wohl jetzt? Hat sie den Ausstieg aus der Depression, dem selbstdestruktiven Verhalten und der Suizidalität geschafft? Ich gehe quer durchs Zimmer zum Schreibtisch, nehme den Arztbericht und trenne die handschriftlich verfasste Notiz davon. Noch ein letztes Mal lese ich ihn durch, bevor 
ich den Abschiedsbrief zerreisse, meinen Abschiedsbrief.

22.10.2012. Dieser Tag sitzt heute noch, nach 8 Jahren, tief in mir. Er hat mein Leben und meine Denkweise geprägt. Durch die Behandlung des Fachteams wurde mir eine zweite Chance auf das Leben gegeben, ein neuer Weg eröffnet, welcher zu diesem heutigen Zeitpunkt geführt hat. Das junge verzweifelte Mädchen seraphina.limacher[at]gmx.ch erscheint mir so fremd und doch so vertraut, zugehö- rig zu meiner Vergangenheit, aber immer präsent in meiner Gegenwart und Zukunft. Sie wird mir im Hinterkopf beistehen und das Selbstvertrauen geben, die richtigen Entscheidungen für meine Patientinnen und Patienten zu treffen.

\section{Literatur}

1 Kant IM. Grundlegung zur Metaphysik der Sitten. Timmermann J, Hrsg. Göttingen: Vandenhoeck \& Ruprecht; 2004, S. 44 